\title{
B cell receptor revision diminishes the autoreactive $B$ cell response after antigen activation in mice
}

\author{
Ying-Hua Wang'1 and Betty Diamond ${ }^{2}$
}

\begin{abstract}
1Department of Microbiology, Columbia University Medical Center, New York, New York, USA. ${ }^{2}$ The Center for Autoimmune and Musculoskeletal Disease, The Feinstein Institute for Medical Research, North Shore Long Island Jewish Health System, Manhasset, New York, USA.
\end{abstract}

\begin{abstract}
Autoreactive $\mathrm{B}$ cells are regulated in the $\mathrm{BM}$ during development through mechanisms, including editing of the $B$ cell receptor (BCR), clonal deletion, and anergy. Peripheral B cell tolerance is also important for protection from autoimmune damage, although the mechanisms are less well defined. Here we demonstrated, using a mouse model of SLE-like serology, that during an autoimmune response, RAG was reinduced in antigen-activated early memory or preplasma B cells. Expression of RAG was specific to antigen-reactive B cells, required the function of the IL-7 receptor (IL-7R), and contributed to maintenance of humoral tolerance. We also showed that soluble antigen could diminish a non-autoreactive antibody response through induction of $B C R$ revision. These data suggest that tolerance induction operates in B cells at a postactivation checkpoint and that $\mathrm{BCR}$ revision helps regulate autoreactivity generated during an ongoing immune response.
\end{abstract}

\section{Introduction}

During B lymphocyte development in the BM, immature B cells expressing self-reactive antigen receptors are tolerized by a variety of mechanisms. Prominent among these are clonal deletion, which purges $B$ cells reactive to self antigens from the repertoire (1), and induction of clonal anergy, which renders autoreactive $B$ cells nonresponsive to $B$ cell receptor (BCR) stimulation $(1,2)$. In addition, secondary variable (diversity) joining $[\mathrm{V}(\mathrm{D}) \mathrm{J}]$ rearrangement mediated by RAG1 and RAG2, which is termed receptor editing and occurs particularly at Ig light chain loci, helps maintain tolerance by modifying the specificity of the BCR $(3,4)$. The fate of individual autoreactive B cells is determined by multiple factors, including the strength of signaling through the BCR, the developmental stage at which BCR engagement by self antigen occurs, whether the self antigen is soluble or membrane bound, and the presence of costimulatory factors, cytokines, or toll-like receptor ligands that can rescue $B$ cells triggered for tolerance induction. Low-affinity autoreactive B cells, B cells that do not encounter self antigen at the immature or transitional stage, and $\mathrm{B}$ cells rescued from deletion enter into the naive, mature, immunocompetent B cell repertoire (5).

For these reasons and because autoreactivity is generated de novo by somatic mutation of Ig variable region (V) genes, tolerance mechanisms continue to operate in the periphery. Even antigen-activated B cells can be subject to tolerance mechanisms. IL- 6 has been reported to mediate tolerance in B cells subject to chronic antigen exposure (6). Antigen-activated B cells can also be blocked from becoming antibody-secreting plasma cells (7). It is controversial whether receptor editing, or receptor revision as it has been termed in mature cells, is a tolerance mechanism

Nonstandard abbreviations used: AID, activation-induced deaminase; APC, allophycocyanin; BCR, B cell receptor; IgH, Ig heavy chain; IL-7R, IL-7 receptor; KLH, keyhole limpet hemocyanin; qPCR, quantitative real-time PCR; Sm, Smith antigen; Tet $^{+}$, tetramer-reactive; $V$, variable region.

Conflict of interest: The authors have declared that no conflict of interest exists. Citation for this article: J. Clin. Invest. 118:2896-2907 (2008). doi:10.1172/JCI35618. for antigen-activated B cells. Several groups have reported the induction of RAG proteins in mature B cells, both in vitro and in vivo $(8,9)$. Indeed, evidence of secondary, heavy chain $V(D) J$ rearrangement has been observed in GC B cells, following exposure to soluble antigen (10). Moreover, RAG-expressing B cells have been observed in the peripheral blood of lupus patients $(11,12)$, the synovial tissue of patients with rheumatoid arthritis (13), and the extrafollicular areas of normal human tonsils (14). Since receptor editing or receptor revision leads to the generation of a novel BCR with a novel antigenic specificity, it may be a potential mechanism for regulating self-reactive $B$ cells during an ongoing immune response.

The pentapeptide DWEYS is a mimetope of dsDNA. When it is part of a decapeptide octamerized on a polylysine backbone (DWEYS-MAP), it can induce a SLE-like serology in the nonautoimmune BALB/c mouse strain, while immunization with the polylysine backbone (MAP-core) alone does not (15). Because we are able to identify the antigen-specific $B$ cells in the spleens of mice immunized with DWEYS-MAP using a fluorochrometagged tetrameric peptide (16), we were able to demonstrate RAG expression and $\lambda$ light chain expression in antigen-activated autoreactive B cells (17). Importantly, we failed to detect RAG expression in antigen-specific B cells in the response to a control peptide mimetope of phosphorylcholine that does not generate autoreactivity, despite provoking a robust anti-phosphorylcholine response. We hypothesized, therefore, that receptor revision occurs during an autoimmune response. We now demonstrate that receptor revision occurs in B cells that appear to have experienced GC maturation and that the RAG-expressing B cells have characteristics of early memory or preplasma cells. The induction of RAG in antigen-activated B cells requires the function of the IL-7 receptor (IL-7R, CD127). We further demonstrate that soluble antigen is able to induce receptor revision in antigenreactive $B$ cells, thus providing the mechanism for induction of RAG. Finally, we show that receptor revision acts as an important mechanism of tolerance that restricts the autoreactivity generated in an ongoing $B$ cell response. 
A

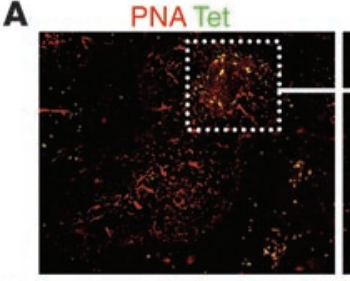

B
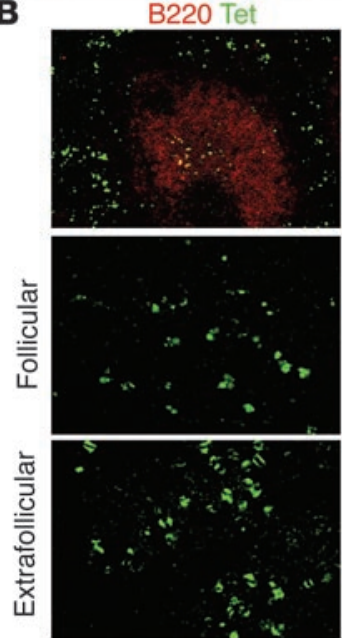

PNA Tet

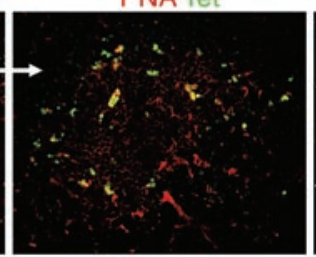

B220 RAG2

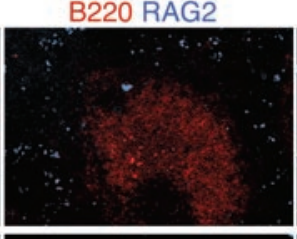

PNARAG2

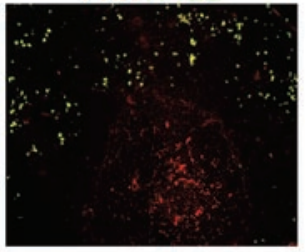

B220 Tet RAG2
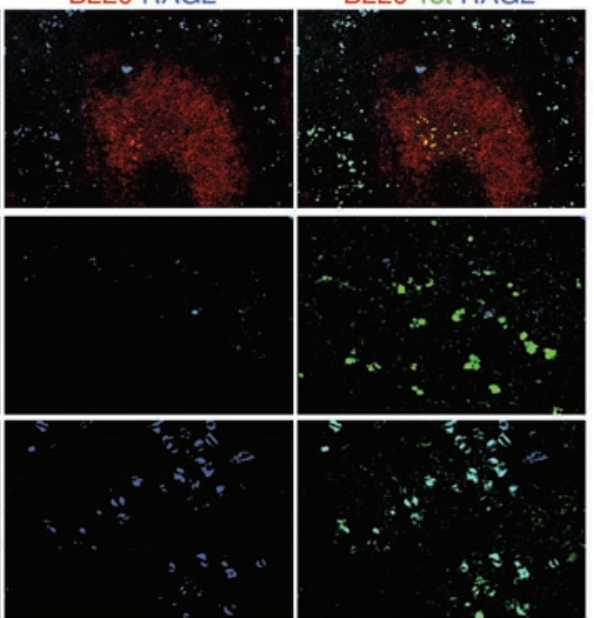

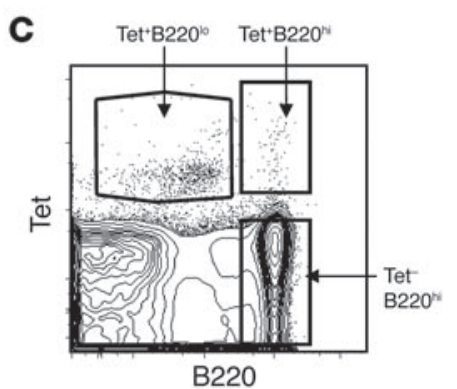

D
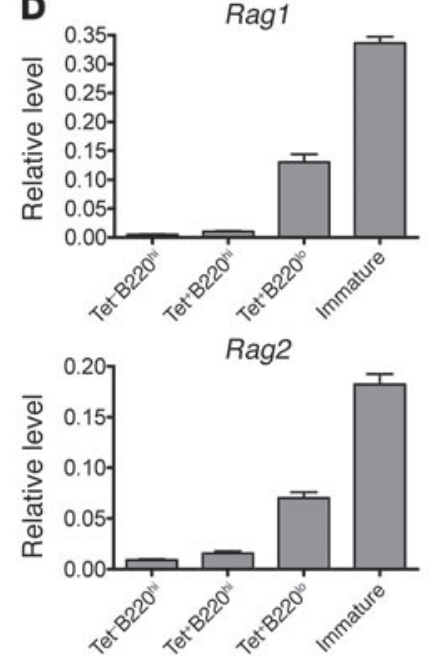

Figure 1

RAG is induced in antigen-reactive early memory/preplasma B cells. (A) Histological staining of spleen sections for peanut agglutinin (PNA) (red) and DWEYS-tetramer (green, left 2 panels) or RAG2 (green, right panel). Original magnification: $\times 100$ (left and right panels); $\times 400$ (middle panel). (B) Histological staining of spleen sections for B220 (red), tetramer binding (green), and RAG2 (blue). RAG2 is coexpressed in extrafollicular tetramer-binding cells. Original magnification: $\times 100$ (top row); $\times 400$ (middle and bottom rows). Three to five mice were used in each group. Four to six pictures were taken for each spleen section. (C) Bivariate plots showing the gates for sorting the specified B cell subsets. (D) qPCR

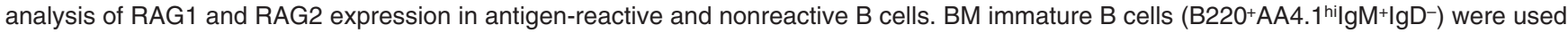
as a positive control. Mice were immunized with DWEYS-MAP in CFA on day 0 and boosted with DWEYS-MAP in incomplete Freund adjuvant on day 7. On day 16, specified B cell subsets were sorted by flow cytometry for preparation of RNA. RNA polymerase 2a (Polr2a) was used as internal control gene. Data (mean \pm SEM) are representative of at least 4 independent experiments. Relative level is defined as the relative expression of mRNA normalized to that of Polr2a.

\section{Results}

$R A G$ is induced in postactivation early memory/preplasma $B$ cells following DWEYS-MAP immunization. BALB/c mice immunized with DWEYS-MAP develop T cell-dependent, anti-DNA/antipeptide, cross-reactive antibody responses $(15,18)$. Using a tetrameric form of this peptide, we are able to detect the tetramer-reactive $\left(\mathrm{Tet}^{+}\right)$ compartment, which is enriched in DNA-reactive B cells $(16,17)$. In a previous study, we reported that RAG expression is detected in a subpopulation of the splenic antigen-reactive compartment within a short time window following immunization with DWEYS-MAP (17). In this study, we sought to further delineate the developmental stage of antigen-reactive RAG-expressing cells and to understand the mechanism and function of receptor editing in an ongoing response.

First, we analyzed spleen sections prepared on day 16 following DWEYS-MAP immunization to determine the location of antigen-reactive and RAG-expressing B cells, at the time we knew them to display maximum RAG expression (17). We used B220 to identify B cell follicles and peanut agglutinin (PNA) to identify GCs. Histologic analysis indicated that there were $\mathrm{Tet}^{+}$cells within GCs, but RAG did not localize to the GCs (Figure 1A). Instead, costaining of spleen sections with antibody to RAG2 and fluorochrome-tagged tetramer demonstrated RAG2 expression in antigen-binding cells in extrafollicular loci but not in antigenbinding cells in follicles or GCs (Figure 1B).

We next tried to ascertain the differentiation state of antigen-reactive, RAG-expressing B cells. Based on the expression of B220, Tet $^{+}$cells can be divided into a major B220 lo subset (around $1 \%$ of B cells) and a minor B220hi subset (around $0.2 \%$ of B cells) (17). Previously, we hypothesized that B220 ${ }^{\text {lo }}$ cells derive from $\mathrm{B} 220^{\mathrm{hi}}$ cells, as the B220 ${ }^{\mathrm{hi}}$ subset included a large percentage of cells expressing the GC marker GL-7 and activation markers, and the $\mathrm{B} 220^{\mathrm{lo}}$ subset included a larger percentage of CD $138^{+}$ plasma cells (17). In order to determine which population expressed RAG, we isolated $\mathrm{Tet}^{+} \mathrm{B} 220^{\text {hi }}$ and $\mathrm{Tet}^{+} \mathrm{B} 220^{\text {lo }} \mathrm{B}$ cells by fluorescence-activated cell sorting (FACS) (Figure 1C) and measured Rag1 and Rag2 mRNA levels by quantitative real-time PCR (qPCR) in these populations. Both RAG1 and RAG2 transcripts were upregulated only in the $\mathrm{Tet}^{+} \mathrm{B} 220^{\text {lo }} \mathrm{B}$ cell population, and the degree of upregulation was approximately $30 \%$ of that seen 
A

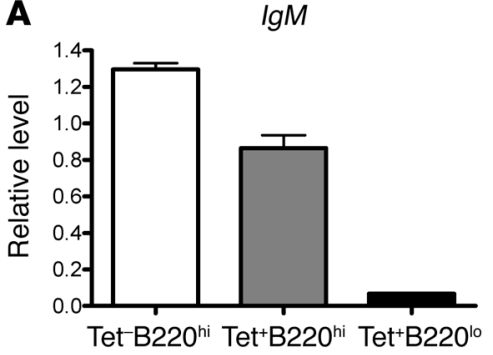

C
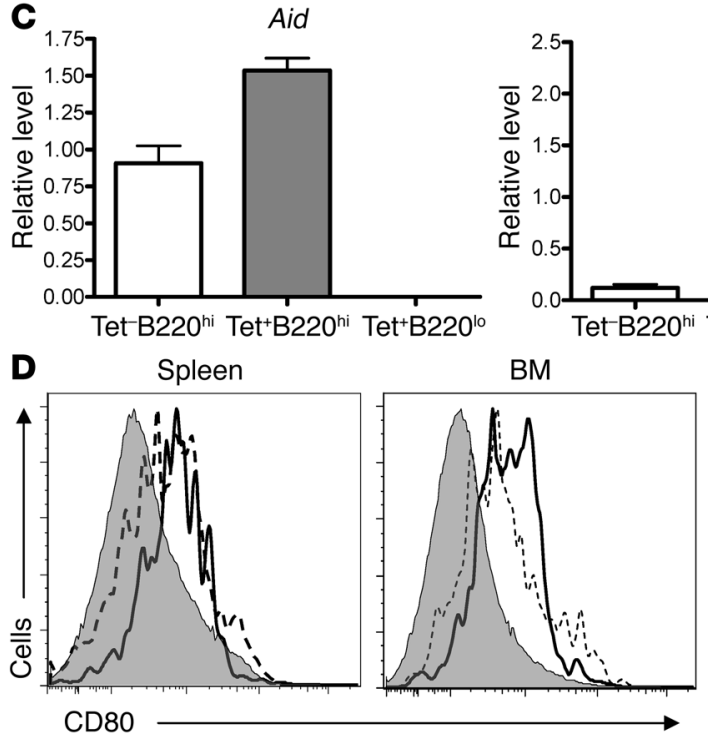

$\lg G 1$

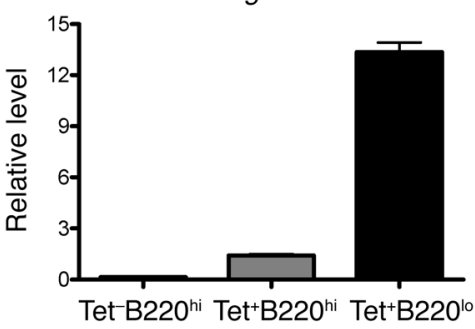

Blimp1

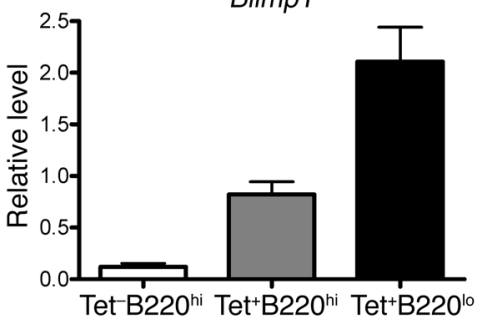

Spleen

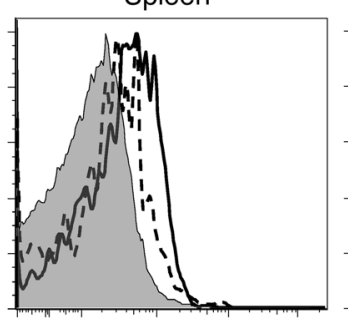

CD95

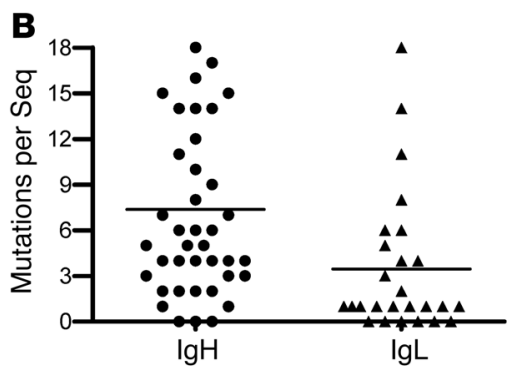

Xbp1

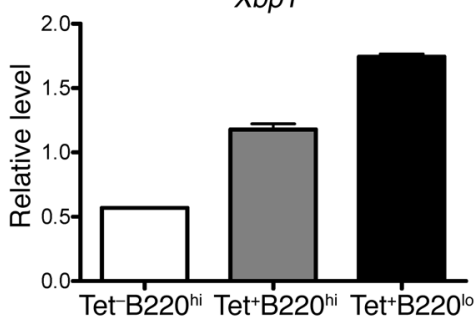

$\mathrm{BM}$

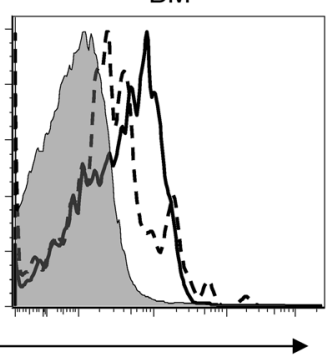

Figure 2

Characterization of antigen-reactive and nonreactive B cells. (A) qPCR analysis of transcripts of $\mu$ and $\gamma 1$ chains in antigen-reactive and nonreactive B cells. (B) Sequence (Seq) analysis of $\mathrm{VH}$ and $\mathrm{V}_{\kappa}$ genes expressed by antigen-reactive B cells. Filled circles and triangles represent the number of point mutations in individual VH genes $(n=41)$ and $\mathrm{V}_{\kappa}$ genes $(n=26)$ expressed by Tet ${ }^{+} \mathrm{B} 220^{\mathrm{lo}}$ cells. (C) qPCR analysis of Aid, Blimp1, and Xbp1 in antigen-reactive and nonreactive B cells. Data (mean \pm SEM) are representative of 3 independent experiments (A and C). ND, not detected. Mice were immunized with DWEYS-MAP in CFA on day 0 and boosted with DWEYS-MAP in incomplete Freund adjuvant on day 7. Cells were sorted on day $16(\mathbf{A}-\mathbf{C})$. (D) Flow cytometry analysis of surface CD80 and CD95 on Tet+B220'o (solid line), Tet+B220hi (dotted line), and Tet-B220 hi (line over shaded area) subsets. Mice were immunized with DWEYS-MAP in CFA on day 0 and boosted with DWEYS-MAP in incomplete Freund adjuvant on days 7 and 56. Spleen and BM cells were prepared 4 days after the second boost for analysis. Representative histogram from 4 to 5 mice was shown.

in immature BM B cells (Figure 1D). Single-cell PCR analysis showed that approximately $30 \%$ of Tet $^{+} \mathrm{B} 220^{\text {lo }} \mathrm{B}$ cells expressed RAG2 transcript (data not shown). This was confirmed by histology analysis of spleen sections and was consistent with flow cytometry results reported previously (17).

In $\mathrm{T}$ cell-dependent antibody responses, $\mathrm{B}$ cells can be activated either to form GCs or to locate in extrafollicular areas and become plasmablasts (19). Because tetramer-binding B cells were detected in both GCs and extrafollicular loci, while RAG ${ }^{+}$cells localized only in extrafollicular sites, we asked whether the extrafollicular antigen-reactive $\mathrm{RAG}^{+}$cells were derived from a $\mathrm{GC}$ response or from an extrafollicular response or both. We, therefore, immunized the mice with DWEYS-MAP and prepared spleens at different time points for histological analysis. On day 5, the peak time of a typical extrafollicular response (20), very few tetramerbinding cells were detected within GCs or in extrafollicular loci. Tetramer-binding cells first appeared within GCs on day 8 and became evident in extrafollicular sites on day 11. Abundant tetramer-binding cells were detected in extrafollicular loci on day 16
(Supplemental Figure 1). A similar result was obtained by flow cytometry analysis, which demonstrated that the Tet ${ }^{+} \mathrm{B} 220^{\text {lo }}$ subset appeared 11 to 12 days after immunization, while Tet $^{+} B 220^{\text {hi }}$ cells were detectable as early as day 8 (data now shown). As mentioned above, our previous studies also demonstrated that GL-7 ${ }^{+}$ cells were predominantly in the B220 hi subset (17).

To characterize the antigen-binding $\mathrm{B}$ cells further, we analyzed $\operatorname{Ig}$ heavy chain $(\mathrm{IgH})$ expression in each subset by qPCR. We found that the $\mathrm{Tet}^{+} \mathrm{B} 220^{\text {hi }}$ subset expressed a high level of IgM heavy chain and a low level of IgG1 heavy chain compared with the $\mathrm{Tet}^{+} \mathrm{B} 220^{\text {lo }}$ subset, which expressed a high level of IgG1 and a low level of IgM (Figure 2A), consistent with the B220 lo subset representing a further stage in differentiation. To determine whether these antigen-reactive cells have indeed experienced GC maturation, we looked for evidence of somatic mutation, a characteristic of GC-matured B cells. We sequenced Ig V genes. IgH V genes of $\mathrm{Tet}^{+} \mathrm{B} 220^{\text {lo }}$ cells were hypermutated (Figure $2 \mathrm{~B}$ ). In addition, most Ig light chain (IgL) V genes of the $\mathrm{Tet}^{+} \mathrm{B} 220^{\text {lo }}$ cells were also mutated, although to a lesser extent (Figure 2B). In contrast, 
A

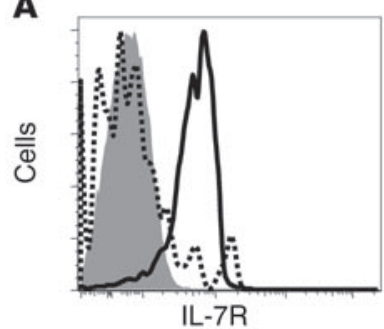

B
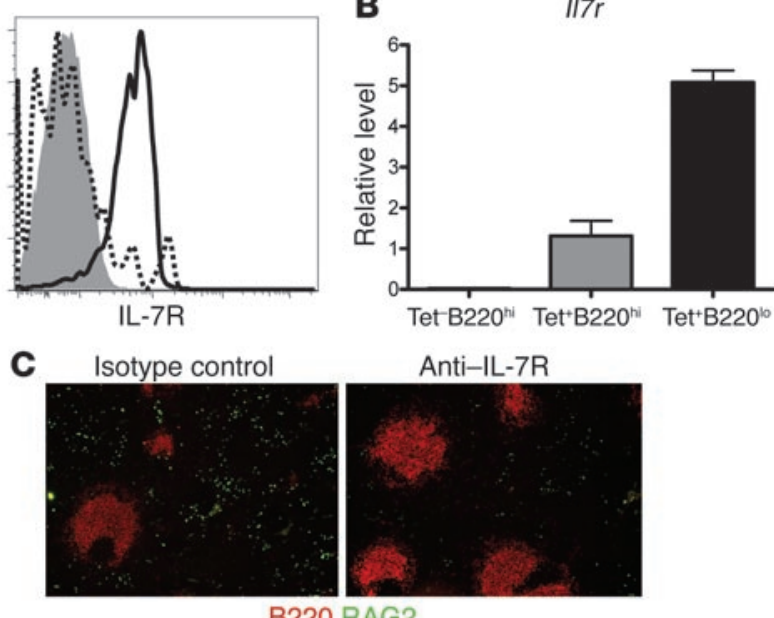

B220 RAG2

D

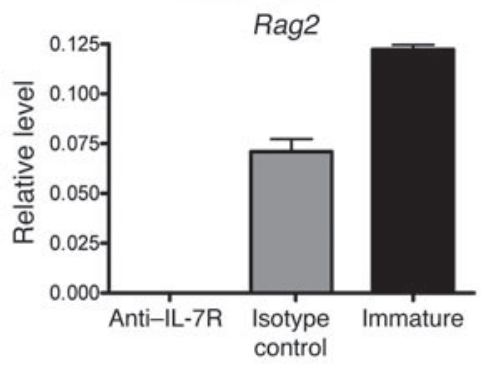

the Ig V genes expressed by the $\mathrm{Tet}^{+} \mathrm{B} 220^{\text {hi }} \mathrm{B}$ cells were primarily unmutated (data not shown). These data suggest that the $\mathrm{Tet}^{+} \mathrm{B} 220^{\text {lo }}$ population has undergone GC differentiation, while the $\mathrm{Tet}^{+} \mathrm{B} 220^{\text {hi }}$ population, which we previously showed included GL- $7^{+}$cells, might be in an early stage of the GC response.

Differentiation of antigen-activated B cells is accompanied by a program of gene expression $(21,22)$. Activation-induced deaminase (AID) is highly expressed in GC B cells and required for both somatic hypermutation and class switch recombination. By qPCR, we observed that AID was upregulated about 2 -fold in Tet $^{+} \mathrm{B} 220^{\text {hi }}$ cells compared with non-antigen-specific Tet ${ }^{-} \mathrm{B} 220^{\text {hi }}$ B cells (Figure $2 \mathrm{C}$ ). While AID expression in the $\mathrm{Tet}^{+} \mathrm{B} 220^{\text {hi }}$ subset was less than in a population of isolated GC B cells (data not shown), it was substantially higher than in naive Tet- $\mathrm{B} 220^{\text {hi }} \mathrm{B}$ cells. In contrast, Aid was undetectable in the $\mathrm{Tet}^{+} \mathrm{B} 220^{\text {lo }}$ population. Blimp1, a transcriptional repressor, and $X b p 1$, a transcriptional activator, are required for plasma cell differentiation. In contrast to AID, expression of these 2 genes was upregulated to a greater extent in the $\mathrm{Tet}^{+} \mathrm{B} 220^{\text {lo }}$ compartment than the $\mathrm{Tet}^{+} \mathrm{B} 220^{\text {hi }}$ compartment, although both $\mathrm{Tet}^{+}$subsets show higher expression than naive $\mathrm{B}$ cells (Figure 2C). These observations suggest that the $\mathrm{Tet}^{+} \mathrm{B} 220^{\text {lo }}$ subset has progressed further through a differentiation pathway than the $\mathrm{Tet}^{+} \mathrm{B} 220^{\text {hi }}$ subset.

We next wanted to determine if the $\mathrm{Tet}^{+} \mathrm{B} 220^{\text {lo }}$ subset was a memory cell population. Memory B cell development is characterized by the expression of certain surface markers. Among these markers, CD80 and CD95 have been shown to be expressed at a higher level in memory than naive B cells (23). In order to determine whether the newly generated $\mathrm{Tet}^{+}$cells develop into memory cells, mice were immunized with DWEYS-MAP as described and boosted 8 weeks later. Four days after the boost, we analyzed the

\section{Figure 3}

DWEYS-MAP induced RAG expression requires IL-7R signaling. (A) Flow cytometry analysis of IL-7R expression on Tet+B220lo (solid line), $\mathrm{Tet}^{+} \mathrm{B} 220^{\text {hi }}$ (dotted line), and Tet-B220 ${ }^{\text {hi }}$ (line over shaded area) subsets. Splenic cells were prepared on day 16 from DWEYS-MAP-immunized mice. The experiment was repeated twice with 5 mice at each time. The $\mathrm{Tet}^{+} \mathrm{B} 220^{\mathrm{lo}}$ cells display the highest increase in the level of IL-7R protein. (B) qPCR of II7r on the specified B cell subsets. Cells from DWEYS-MAP-immunized mice were sorted by flow cytometry on day 16. Data (mean \pm SEM) represent 1 of 3 independent experiments. $\mathrm{Tet}^{+} \mathrm{B} 22 \mathrm{O}^{\mathrm{lo}}$ cells show the greatest increase in II7r mRNA. (C) Histological staining of spleen sections for B220 (red) and RAG2 (green). Mice were immunized with DWEYS-MAP on day 0 , boosted on day 7 , and treated with anti-IL-7R-blocking antibody or with isotype control on days 8,11 , and 14 . Spleens were harvested on day 16 . Three mice were included in each group. A minimum of 6 pictures were taken for each spleen. The experiment was repeated twice. Spleens from mice treated with anti-IL-7R antibody showed decreased RAG2 expression (green). Follicles are identified by B220 (red). Original magnification: $\times 50$. (D) qPCR analysis of Rag2 in Tet ${ }^{+} B 220^{\circ}$ cells from DWEYS-MAPimmunized mice treated with anti-IL-7R Ab or with isotype control. The procedure for immunization and administration of antibodies was described in C. Cell sorting was done on day 16 , following immunization. Data (mean \pm SEM) are representative of 3 independent experiments. Rag2 mRNA is undetectable in mice treated with anti-IL7R antibody. For all these experiments, the mice were immunized with DWEYS-MAP in CFA on day 0 and boosted with DWEYS-MAP in incomplete Freund adjuvant on day 7.

antigen-reactive cells and nonreactive B cells by flow cytometry. In the spleen, both CD80 and CD95 were upregulated in the B220lo and B220hi Tet-binding compartments, as compared with non-antigen-reactive naive $\mathrm{B}$ cells (Figure 2D), with expression on $\mathrm{Tet}^{+} \mathrm{B} 220^{\text {lo }}$ cells higher than on the $\mathrm{Tet}^{+} \mathrm{B} 220^{\text {hi }}$ subset. Thus, the $\mathrm{Tet}^{+} \mathrm{B} 220^{\text {lo }}$ population had features of memory B cells. Because antigen-specific memory B cells appear in the BM after immunization $(17,24)$, we also analyzed the BM Tet-binding cells and observed memory B cell markers on Tet $^{+}$cells (Figure 2D). To access memory function in Tet $^{+}$cells, we isolated lymphocytes from spleens of immunized mice, either including or excluding $\mathrm{Tet}^{+}$cells, and transferred them into mice with a mutation in the membrane exon of the Ig $\mu$ heavy chain that lack membrane IgM and therefore fail to produce mature B cells ( $\mu$ MT mice) (25). CD $138^{+}$plasma cells were excluded before transfer. When we boosted the recipient mice, a memory response was induced when the Tet $^{+}$cells were present but not in the absence of $\mathrm{Tet}^{+}$ cells (Supplemental Figure 2). Tet $^{+}$cells were, therefore, capable of differentiating into antibody-secreting cells upon rechallenge with antigen and, therefore, included memory B cells.

In conclusion, the $\mathrm{Tet}^{+} \mathrm{B} 220^{\text {lo }} \mathrm{B}$ cells identified on day 16 after immunization were early memory/preplasma B cells that were isotype switched, hypermutated, and exiting the GC reaction, while the $\mathrm{Tet}^{+} \mathrm{B} 220^{\text {hi }}$ cells represented an earlier stage of differentiation, perhaps GC cells.

Next, we wanted to confirm that RAG was expressed in mature $\mathrm{B}$ cells, because the literature on this topic is quite controversial. We transferred BALB/c naive spleen cells to SCID mice, waited for 2 weeks for transitional B cells to die or mature, and immunized with DWEYS-MAP or MAP-core. RAG was expressed in the SCID spleens after immunization with the DWEYS-MAP but not in 

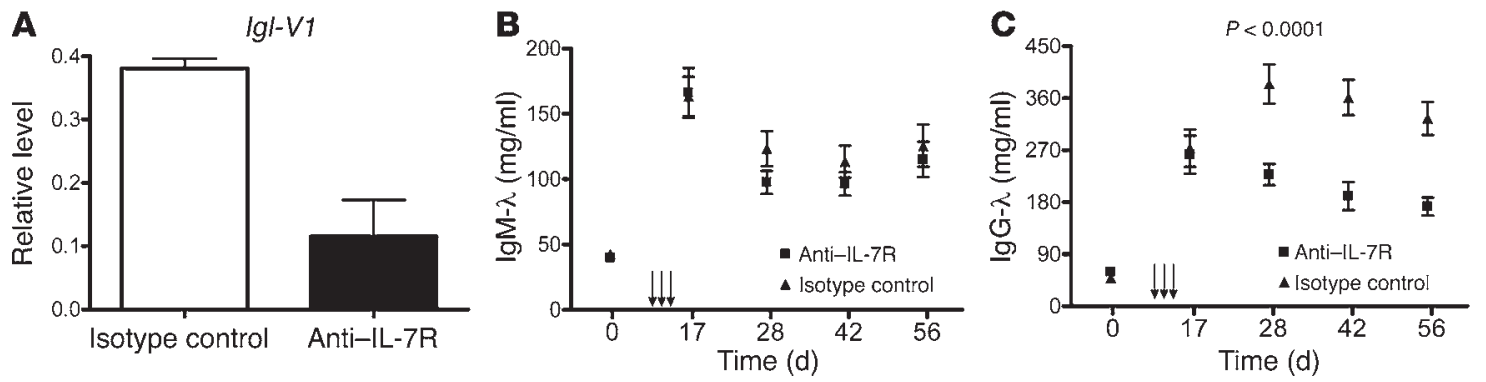

\section{Figure 4}

Inhibition of RAG leads to decrease of $\lambda$ chain expression. (A) qPCR for $\lambda$ chain expression (Igl-V1) on Tet ${ }^{+} B 220^{\circ}$ cells. Mice were immunized with DWEYS-MAP and treated with anti-IL-7R antibody or isotype control as described in Figure 3 , and spleen cells were sorted on day 16 . The experiment was performed twice. (B and C) ELISA of serum levels of $\lambda$ chain associated with IgM (B) or IgG (C) from immunized mice. Arrows indicate the time of injection of anti-IL-7R antibody or isotype control on days 8,11 , and 14. Data are the mean \pm SEM of 5 mice in each group. The $P$ value was determined by 2-way ANOVA.

naive or MAP-core immunized spleens (17) (data not shown). In order to rule out absolutely that the appearance of antigen-binding $\mathrm{RAG}^{+}$cells in DWEYS-MAP-immunized mice was caused by contamination of the transferred cells with immature $\mathrm{B}$ cells or pro- or pre-B cells, we isolated naive mature (AA4.1- $\mathrm{HSA}^{\mathrm{lo}}$ ) lymphocytes from $B A L B / c$ mice and adoptively transferred them to $R A G 2^{-/-}$mice. In this experiment, immature B cells were excluded from transfer, and RAG transcript was undetectable in the sorted cells prior to transfer (data not shown). In addition, RAG transcript was not detected in the spleen after splenocytes were transferred to $R A G 2^{-/-}$mice prior to immunization (data not shown). When we immunized the recipient mice with DWEYS-MAP, we again detected both RAG1 and RAG2 expression in the Tet ${ }^{+}$B220lo subset (Supplemental Figure 3). Since there are no immature B cells exiting from the $\mathrm{BM}$ in $R A G 2^{-/-}$mice, the $\mathrm{B}$ cell repertoire in the recipient mice was composed exclusively of mature $B$ cells. These data demonstrate that the appearance of $\mathrm{RAG}^{+} \mathrm{B}$ cells in the spleen of DWEYS-MAP-immunized mice is not a result of contamination by immature B cells or BM emigrants. Rather, RAG is reinduced in mature immune-competent B cells in an antigen-dependent manner.

$R A G$ expression and receptor revision in antigen-reactive $B$ cells is dependent on $I L-7 R$ function. It has been reported that expression of RAG proteins, or their functionality, in developing B cells is dependent on IL-7R signaling (26-30). Therefore, we wanted to know whether IL-7R is also involved in the regulation of RAG expression in the spleen of DWEYS-MAP-immunized mice. First, we analyzed the expression of IL-7R in the antigen-reactive $B$ cells of immunized mice. Both flow cytometry for IL-7R protein expression and qPCR for $I l 7 r$ mRNA on isolated $\mathrm{Tet}^{+} \mathrm{B} 220^{\mathrm{hi}}$ and $\mathrm{Tet}^{+} \mathrm{B} 220^{\text {lo }} \mathrm{B}$ cells showed that IL-7R was most highly upregulated in the $\mathrm{Tet}^{+} \mathrm{B} 220^{\text {lo }}$ population (Figure 3 , A and B). Naive Tet-B220 hi $\mathrm{B}$ cells showed little expression of IL-7R protein or $I l 7 r$ mRNA. Once we established specific expression of IL-7R on Tet ${ }^{+} B 220^{\text {lo }}$ $B$ cells, we asked whether IL-7R signaling was critical for RAG expression. Mice were immunized with DWEYS-MAP on day 0 and boosted on day 7. Either anti-IL-7R-blocking antibody or isotype control antibody was administered to the mice on days 8 , 11 , and 14. Histological analysis of spleen sections showed that the expression of RAG2 was substantially inhibited by treatment of the mice with anti-IL-7R-blocking antibody (Figure 3C). qPCR analysis on sorted cells demonstrated an inhibition of RAG2 at the transcriptional level, as we were unable to detect the RAG2 transcript in the $\mathrm{Tet}^{+} \mathrm{B} 220^{\text {lo }}$ compartment of anti-IL-7R-treated mice (Figure 3D). Importantly, there was no toxicity associated with the anti-IL-7R antibody, as the $\mathrm{Tet}^{+} \mathrm{B} 220^{\text {lo }}$ cells remained positive for expression of IL-7R, even in mice given anti-IL-7R antibody (Supplemental Figure 4). Thus, the anti-IL-7R antibody did not cause the elimination of cells expressing IL-7R. Because IL-7 has been shown to regulate survival and homeostasis of naive and memory $T$ cells $(31,32)$, we wondered whether blocking the IL-7R pathway might affect helper $\mathrm{T}$ cell function and thus impair the GC response. We, therefore, stained the spleen sections from DWEYS-MAP-immunized mice treated with antiIL-7R or isotype control antibody for GCs and antigen-binding B cells. Anti-IL-7R neither caused an apparent defect in GC formation nor altered the frequency of tetramer-binding cells (data not shown). Furthermore, when the $\operatorname{IgH} \mathrm{V}$ gene sequences were analyzed, the mutation rate of $\mathrm{Tet}^{+} \mathrm{B} 220^{\mathrm{lo}}$ cells was not changed by anti-IL-7R treatment (Supplemental Figure 4), suggesting that there was no defect in the GC response. These observations suggest that the transient blocking of IL-7R did not impair helper $\mathrm{T}$ cell function and the GC response, consistent with the observation that IL-7R is downregulated on T cells upon TCR activation $(31,33)$, Taken together, the reexpression of RAG in the antigen-activated $B$ cells occurs in the context of an adequate $T$ cell response and requires the function of IL-7R on B cells.

Repeated variable joining rearrangement eventually leads to exhaustion of the recombination potential at the $\kappa$ chain $V$ gene locus and expression of a $\lambda$ light chain. Thus, one molecular signature of receptor revision would be an increase in $\operatorname{Ig} \lambda^{+}$cells, and occasionally, coexpression of IgK and $\operatorname{Ig} \lambda$ light chains (34). In our previous study, we showed that $\lambda$ light chain is upregulated in the $\mathrm{Tet}^{+} \mathrm{B} 220^{\text {lo }}$ cells (17), and a significant portion of $\mathrm{Tet}^{+} \mathrm{B} 220^{\text {lo }}$ cells are $\lambda / \kappa$ double positive in DWEYS-MAP-immunized mice (17). Although $\lambda$ light chain is detected in the newly activated $\mathrm{Tet}^{+} \mathrm{B}$ cells, when we generated antigen-specific hybridomas from immunized mice, we failed to identify any antibody-secreting cell that expresses $\lambda$ light chain (35). Similarly, we detected no serum $\lambda^{+}$antibody binding to DWEYS peptide using ELISA (data not shown). Thus, the expression of $\lambda$ light chain is due to receptor revision, rather than positive selection, and inhibition of receptor revision should inhibit $\lambda$ chain expression. We, therefore, analyzed the expression of $\lambda$ chain in the presence or absence 
A

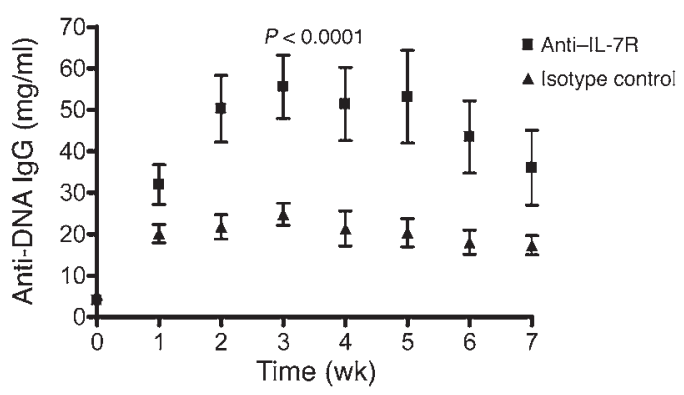

\section{B}

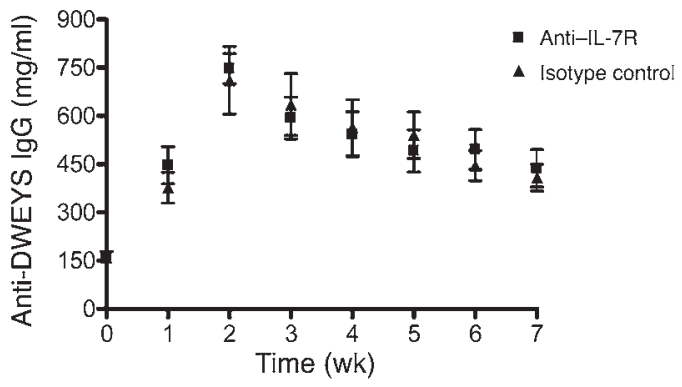

of antibody to IL-7R. qPCR analysis demonstrated that expression of mRNA of the $\lambda$ light chain ( $I g l-V 1)$ in Tet ${ }^{+} \mathrm{B} 220^{\text {lo }}$ population was markedly decreased in mice treated with anti-IL-7Rblocking antibody (Figure 4A).

To demonstrate that receptor revision was occurring selectively in antigen-activated B cells, we measured the serum levels of secreted $\operatorname{IgM} \lambda$ and $\operatorname{IgG} \lambda$ antibodies using ELISA. $\operatorname{IgM} \lambda$ levels were not affected by the treatment with the anti-IL-7R antibody (Figure 4B); however, IgG $\lambda$ levels were significantly decreased in mice treated with anti-IL-7R antibody (Figure 4C). Since the total serum level of IgG was not altered by the treatment with anti-IL-7R antibody (Supplemental Figure 5), we concluded that the decreased expression of $\lambda$ light chain is due to inhibition of receptor revision in antigen-specific $B$ cells that are undergoing or have undergone isotype switching.

Receptor revision is a mechanism to regulate an autoantibody response. The observation that RAG protein was reexpressed in the antigen-experienced, early memory/preplasma cell subpopulation led us to hypothesize that receptor revision may play a role in the regulation of autoreactivity induced by antigen challenge, and the ability of anti-IL-7R-blocking antibody to inhibit RAG expression allowed us to address this question. We immunized $\mathrm{BALB} / \mathrm{c}$ mice with DWEYS-MAP and administered anti-IL-7R or isotype control antibody as described. The mice were bled at the specified days after immunization. In order to investigate the memory response, these mice were boosted 8 weeks after the primary immunization and bled weekly thereafter. We found that in the primary response, the production of IgM antipeptide and anti-dsDNA antibodies was not affected by anti-IL-7R treatment; titers of anti-DNA IgG, but not antipeptide IgG, were modestly increased in anti-IL-7R-treated mice (Supplemental Figure 6). During the memory response, we observed a greater increase in serum anti-DNA IgG titers in mice treated with antiIL-7R-blocking antibody (Figure 5A). The IgG memory response to peptide was not changed by anti-IL-7R treatment (Figure 5B). These results demonstrate that receptor revision attenuates the autoantibody production arising in the course of a response to

\section{Figure 5}

Receptor revision contributes to tolerance maintenance in peptideinduced autoimmunity. BALB/c mice were immunized with DWEYS-MAP peptide and administered anti-IL-7R antibody or isotype control as described in Figure 3. Eight weeks after the primary immunization, the mice were boosted one more time with DWEYS-MAP in incomplete Freund adjuvant. Anti-dsDNA (A) and antipeptide (B) IgG serum antibodies were measured by ELISA after the boost. Data are the mean \pm SEM of 5 mice in each group, representative of 2 independent experiments. The $P$ value was determined by 2 -way ANOVA.

foreign antigen, and the inhibition of receptor revision leads to increased expression of autoreactivity.

Soluble antigen induces $R A G$ expression and receptor revision. Because RAG is induced in autoreactive, early memory/preplasma B cells but not in the equivalent compartment in mice immunized with the non-self 10-2 peptide (17), we speculated that engagement with antigen, in this case, with dsDNA outside the GC environment, may be essential for RAG induction. In order to test this hypothesis, we adoptively transferred naive splenocytes into the $R A G 2^{-/-}$mice and immunized the recipient mice with DWEYS-MAP 3 weeks later. From day 9 to day 15 following immunization, we treated these mice with active or heatinactivated DNase I daily, and analyzed the expression of RAG in antigen-reactive cells on day 16 . The purpose of the adoptive transfer was to exclude the presence of new BM emigrants, some of which express RAG. Treatment with active DNase I resulted in a reduction of plasma DNA by approximately 50\% (data not shown). qPCR analysis showed that the expression of RAG in the Tet ${ }^{+} \mathrm{B} 220^{\text {lo }}$ compartment from DNase I-treated mice was markedly decreased compared with the control group (Figure $6 \mathrm{~A})$. This result suggests that dsDNA in the host is important for RAG induction.

To address definitely whether antigen can actually induce RAG expression and receptor revision during an ongoing immune response, we analyzed 10-2 peptide-immunized mice. Originally identified as a mimetope of phosphorylcholine (36), immunization with 10-2 peptide does not generate an autoreactive $B$ cell response or induce RAG expression by itself (17). BALB/c mice were immunized with 10-2-keyhole limpet hemocyanin (10-2-KLH). On days 13,14 , and 15 following immunization, soluble 10-2-BSA or BSA was administered and splenocytes were examined for RAG induction on day 16. A different protein carrier was used for administration of soluble antigen in order to address specifically the anti-10-2 response.

qPCR analysis of whole spleen cells showed that RAG1 and RAG2 were upregulated by 2 - to 3 -fold by treatment with 10-2-BSA compared with treatment with BSA alone (Figure 6B). Treatment with 10-2-BSA also resulted in an increase of Ig $\lambda$ transcript in antigen-reactive B cells (Figure 6C). Finally, 10-2-BSA administration led to a significant increase of IgG-associated $\lambda$ light chain compared with BSA treatment, another measure of receptor revision (Figure 6D). $\lambda$ light chain associated with IgM was not affected by the treatment with 10-2-BSA (Figure 6D). These results indicate that soluble antigen induced receptor revision in antigen-activated B cells.

Receptor revision reduces the serum antibody response. Next we asked whether induction of receptor revision alters the production of 10-2-specific antibody. Because soluble antigen has been shown to induce apoptosis in GC B cells and reduce memory B cell gener- 

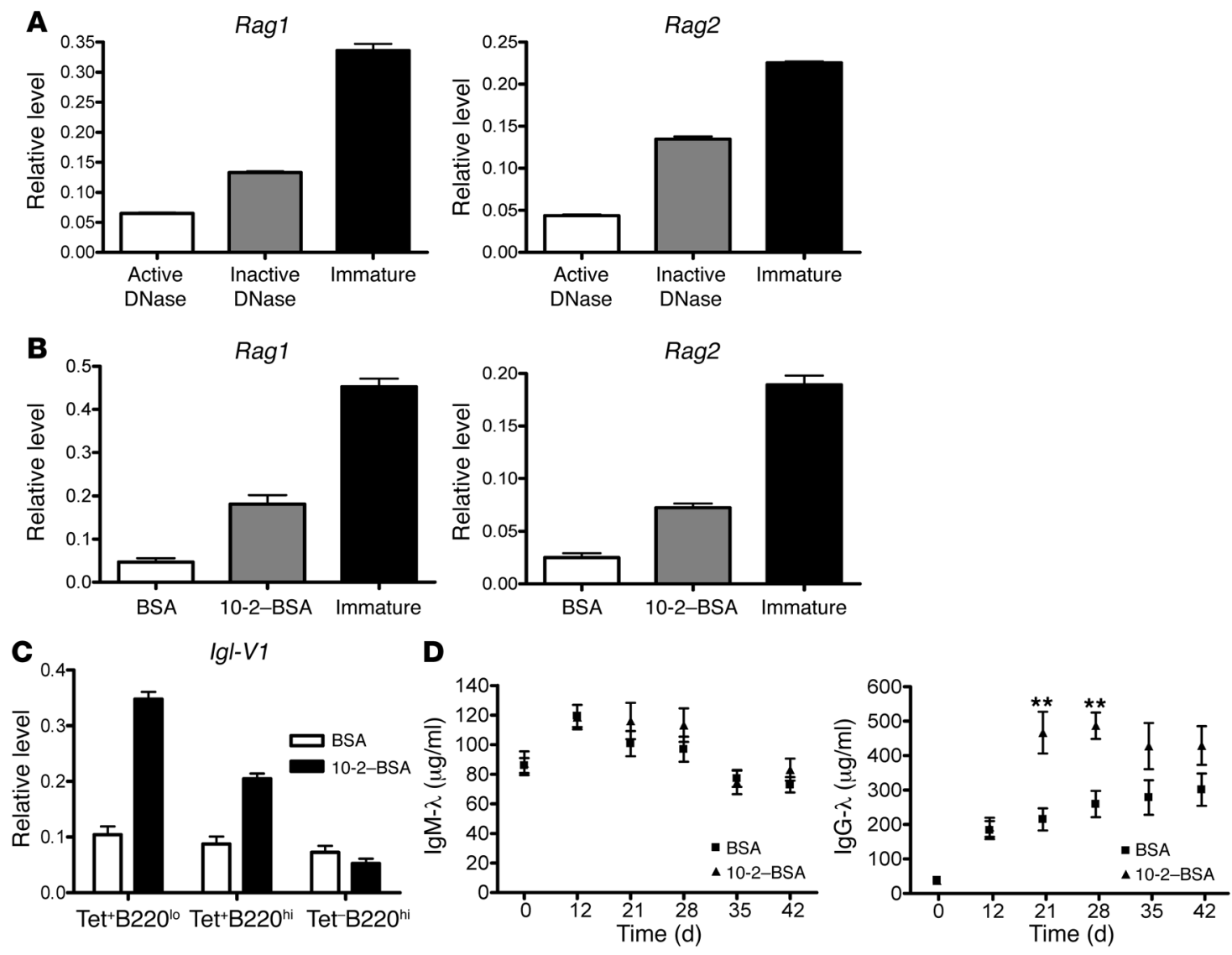

\section{Figure 6}

Soluble antigen induces RAG and receptor revision in the spleen of immunized mice. (A) Expression of RAG in DWEYS tetramer-binding cells requires the presence of circulating dsDNA in the plasma. BALB/c naive splenocytes were adoptively transferred to Rag $2^{-/-}$mice. Three weeks later, the recipient mice were immunized with DWEYS-MAP as described in Figure 3. From day 9 through day 15 following immunization, $500 \mu \mathrm{g}$ active or heat-inactivated bovine DNase I were administered to the mice. Tetramer-reactive cells were sorted on day 16, and Rag1 and Rag2 were analyzed by qPCR. (B) Soluble 10-2-BSA induces RAG in the spleen of mice immunized with 10-2-KLH. (C and D) Receptor revision is induced in antigen-reactive $B$ cells by soluble 10-2-BSA. BALB/c mice were immunized with 10-2-KLH in CFA on day 0 and boosted with 10-2-KLH in incomplete Freund adjuvant on day 7 . On days 13, 14, and 15, $1 \mathrm{mg}$ of 10-2-BSA or BSA were administered. qPCR analysis for the mRNA of $\lambda$ chain $(\mid g /-V 1)$ was done on 10-2-tetramer reactive cells, and nonreactive naive B cells were sorted on day 16 (C). Data (mean \pm SEM) are representative of 2 independent experiments. Serum levels of $\lg \lambda$ were measured by ELISA (D). Five mice were included in each group. The experiment was repeated twice. ${ }^{* \star} P<0.01,2$-tailed Student's $t$ test.

ation $(10,27,37)$, it is not sufficient to compare antibody levels in mice treated with 10-2-BSA and BSA. Since anti-IL-7R antibody can inhibit RAG expression and receptor revision without causing apoptosis, we administered anti-IL-7R or isotype control antibody to mice treated with soluble antigen and measured serum antibody production. The serum level of anti-10-2 IgG, but not IgM, was significantly increased in anti-IL-7R-treated mice, compared with the mice treated with isotype control antibody (Figure 7). Production of antibody against the carrier protein, KLH, was not affected by the treatment with soluble antigen (Supplemental Figure 7), demonstrating that this change occurred in an antigenspecific manner. It is also noteworthy that the levels of anti-10-2 antibody in soluble antigen-treated mice were lower than in BSAtreated mice even when anti-IL-7R was present (Figure 7). This may be due to incomplete inhibition of receptor revision or to the induction of apoptosis by soluble antigen in some antigenreactive $\mathrm{B}$ cells, as previously reported $(10,37,38)$. These data demonstrate that soluble antigen can induce tolerance in anti- gen-reactive B cells during an ongoing immune response through the induction of receptor revision.

\section{Discussion}

Although the naive B cell repertoire is largely purged of selfreactive clones through mechanisms of central tolerance, it is clear that some autoreactive B cells, especially those with low affinity to self antigen, escape central tolerance and mature in the periphery. Early evidence of peripheral tolerance comes from studies using double transgenic mice in which the Ig transgenic $\mathrm{B}$ cells do not encounter self antigen until they exit the BM. In peripheral lymphoid organs, autoreactive B cells are deleted or rendered anergic when they encounter either membrane-bound self antigen or soluble self antigen, respectively $(39,40)$. These studies demonstrate tolerance susceptibility following exodus from the BM. However, it is clear that tolerance must operate in the periphery also, although few mechanisms have been elucidated. In the past few years, a series of peripheral tolerance 

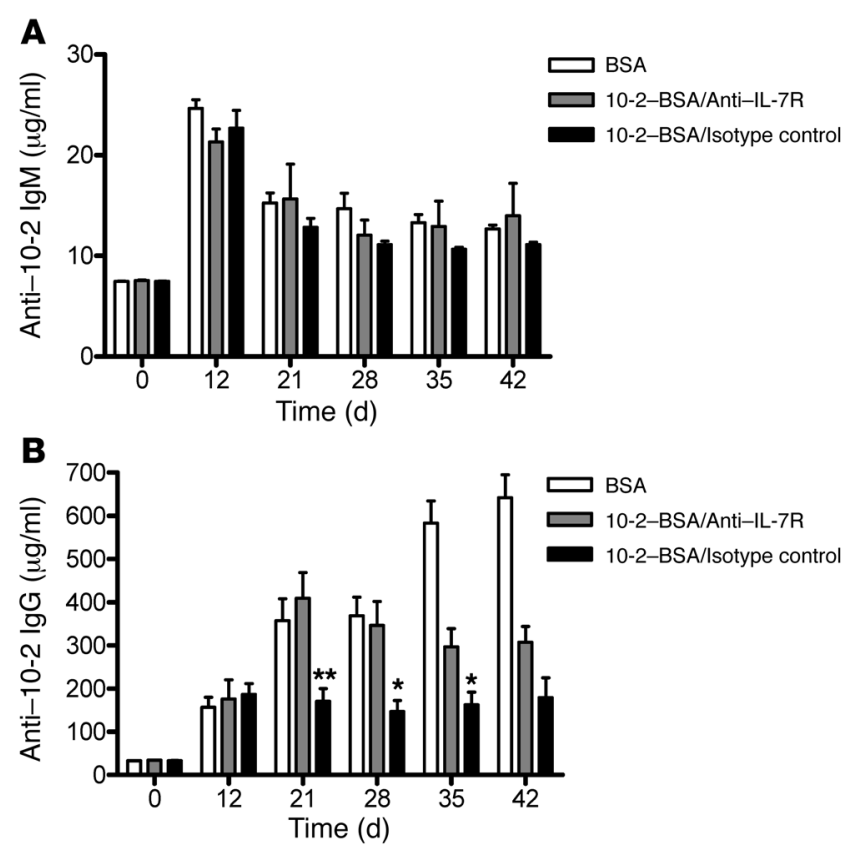

checkpoints have been described. In a study analyzing tolerance induction in human B cells, Tsuiji et al. reported that although up to $20 \%$ of mature naive B cells express antibodies with self reactivity, these $B$ cells are excluded from the repertoire during the transition from IgM naive to IgM memory B cells before the onset of somatic hypermutation (41), implying that tolerance induction is operative in the immunocompetent, naive B cell repertoire in humans also.

William et al. reported that 2 checkpoints exist in the periphery to regulate rheumatoid-factor (RF) expressing autoreactive $B$ cells in mice after exposure to antigen (42). While these cells expand and undergo somatic mutation in the autoimmune MRL/lpr background in a non-autoimmune host, they neither clonally expand nor differentiate into antibody-secreting cells. Tolerance was also reported to operate at a preplasma cell stage in anti-Smith antigen (anti-Sm) IgH Tg B cells (43). Anti-Sm B cells are present at a high frequency in normal mouse spleen and $\mathrm{BM}$ and express the plasma cell marker CD138. However, these cells do not differentiate into antibody-secreting cells in a nonautoimmune strain. Regulation of anti-Sm B cells occurs before the expression of Blimp1, the transcription repressor required for plasma cell differentiation (43). Although it is also observed that anti-Sm B cells have higher turnover rates than B cells not binding $\mathrm{Sm}$, clonal deletion cannot be the only mechanism for tolerance induction, since a high percentage of anti-Sm B cells are still present in the mice. It was determined that IL- 6 secreted by macrophages and dendritic cells helps maintain B cell tolerance in this model (6).

GCs are dynamic microenvironments for B cell differentiation after antigen stimulation. Within GCs, activated B cells rapidly proliferate and undergo somatic hypermutation in their Ig genes. $B$ cells with increased affinity to the eliciting antigen are positively selected and clonally expand. Random $V$ gene mutation, will also often give rise to BCRs that are reactive to self antigen $(15,44,45)$. In normal situations, these autoreactive $\mathrm{B}$ cells are largely prevented from producing autoantibodies and from entering a memory

\section{Figure 7}

Soluble antigen reduces the humoral response by induction of receptor revision. BALB/c mice were immunized and treated with BSA or 10-2-BSA as described in Figure 6. Mice treated with 10-2-BSA were divided into 2 groups. On days 12 and 15, one group was administered with $1 \mathrm{mg}$ of anti-IL-7R antibody, the other group with isotype control antibody. Mice were bled weekly for serum collection. Anti-10-2 IgM (A) and IgG (B) serum antibodies were measured by ELISA. Data are the mean \pm SEM of 5 mice in each group, representative of 2 independent experiments. ${ }^{* *} P<0.01,{ }^{*} P<0.05,2$-tailed Student's $t$ test. The $P$ values show the comparison of the anti-IL-7R-treated group to the group treated with isotype control antibody.

cell compartment (46). Newly formed hapten-specific post-GC B cells were demonstrated to pass through a window of tolerance susceptibility, evidenced by their functional inactivation or deletion following exposure to hapten conjugated to a carrier not recognized by primed $\mathrm{T}$ helper cells (47). In vivo studies confirmed a postactivation checkpoint for B cells. During an ongoing primary immune response in mice, injection of soluble antigen induced extensive apoptosis of GC light zone B cells $(10,37,38)$. This B cell death was antigen specific.

Debate has raged over the question of receptor revision. RAG is expressed in pro-, pre-, and autoreactive immature B cells in the BM and downregulated in naive mature B cells (48). It has been reported that immunization can induce the expression of RAG proteins in peripheral lymphoid organs $(8,9)$. However, the developmental stage of the RAG-expressing B cells was not characterized in these studies. Later studies suggested that the $\mathrm{RAG}^{+}$cells in the spleen of immunized mice were recent emigrants from BM. While their number increased following immunization, they were antigen-independent and were recruited from the BM by adjuvant alone $(49,50)$. These observations suggested that RAG expression in spleen cells was a function of an increased population of immature B cells. In contrast to this theory, RAG was induced in isolated $\mathrm{IgD}^{+}$mature $\mathrm{B}$ cells by stimulation with LPS and various cytokines in vitro $(9,26)$. In human peripheral blood, RAG was found to be expressed in a portion of postswitch ( $\operatorname{IgG}$ ) memory B cells (14). In addition, receptor revision of $\mathrm{IgH} \mathrm{V}$ genes was observed in $\mathrm{IgD}^{+}$tonsil cells and led to the production of hybrid heavy chain $\mathrm{V}$ genes (51). These observations underscore the continuing uncertainty regarding the expression and function of RAG in peripheral B cells. Furthermore, B cells expressing RAG have also been observed outside of GCs in immunized mice (52) and in extrafollicular areas of human tonsil (53). Detection of light chain rearrangement products in RAG-expressing B cells in the periphery clearly suggested that receptor editing or revision may occur in B cells beyond the immature stage $(9,54-56)$. In the periphery, it was reported that RAG is turned off by BCR cross-linking $(33,57)$. However, it is important to note, that these studies were not performed on B cells that experienced antigen challenge.

To monitor the expression of RAG during B cell development, GFP reporter mice have been generated (58-60). Mice in which GFP was expressed under a RAG2 promoter showed no increase in RAG-expressing B cells following immunization (58). Antigenspecific B cells were not analyzed in these studies, and it would have been difficult to observe an overall change in the number of RAG-expressing B cells if the increase in RAG expression was limited to antigen-specific cells. Immunization of mice having a 
fusion gene of RAG2 and GFP replacing the endogenous RAG2 gene locus led to the appearance of GFP in a subset of splenic B cells, approximately $25 \%$ of which expressed GL-7, a marker for GC B cells, although also a marker of immature B cells (59). Thus, studies in these mice did not provide definitive evidence for or against receptor revision, although it was reported that RAG-expressing B cells were detected in $R A G 2^{-/-}$mice that were adoptively transferred with $\mathrm{RAG}^{+/+} \mathrm{BM}$ cells but not with $\mathrm{RAG}^{+/+}$ splenocytes (49).

We have previously reported that immunization with a peptide mimetope of dsDNA (DWEYS-MAP) induces a SLE-like serology in the non-autoimmune BALB/c mouse strain (15). The antibody response is $\mathrm{T}$ cell dependent (18). In order to investigate the DNAreactive $B$ cells participating in the response, we developed a methodology using a peptide tetramer to detect antigen-binding B cells in spleens of immunized mice. The tetramer binding subset is enriched for B cells reactive to dsDNA (16). We immunized BALB/c mice harboring the gene for the RAG2:GFP fusion protein with DWEYS-MAP and observed that approximately $20 \%-50 \%$ of antigen-reactive B cells in the spleen express GFP 16 days after antigen exposure (17). Expression of RAG is antigen dependent and is not observed in mice immunized with a control peptide. The adoptive transfer experiment demonstrated that RAG is de novo induced in mature B cells rather than a consequence of contamination with pro-, pre-, or immature B cells. RAG is specifically induced in the $\mathrm{Tet}^{+} \mathrm{B} 220^{\text {lo }} \mathrm{B}$ cells that express predominantly $\mathrm{V}$ gene mutated and isotype switched antibody.

Analysis of gene expression patterns and surface markers in the antigen-reactive compartments and in naive non-antigen-specific $\mathrm{B}$ cells suggests that the $\mathrm{Tet}^{+} \mathrm{B} 220^{\mathrm{lo}}$ cells are newly generated early memory/preplasma B cells that may derive from the $\mathrm{Tet}^{+} \mathrm{B} 220^{\mathrm{hi}}$ cells, consistent with the results obtained by flow cytometry analysis of antigen-activated B cells in our previous report (17) and by other groups (24). These B cells appear to have matured in a GC environment as they display features of a GC-matured response, heavy chain class switching and somatic hypermutation. Furthermore, we detected antigen-specific cells within GCs. Importantly, histological analysis demonstrated that antigen-reactive B cells first appear within GCs on day 8 following immunization. They are not detectable at extrafollicular loci until day 11. We, therefore, believe that the extrafollicular antigen-reactive $\mathrm{B}$ cells have matured through a GC response.

RAG expression can be identified in antigen-binding B cells only in extrafollicular loci, and receptor revision occurs only in the $\mathrm{Tet}^{+} \mathrm{B} 220^{\text {lo }}$ cells. IL-7R was upregulated in the $\mathrm{Tet}^{+} \mathrm{B} 220^{\text {lo }}$ population also, and the expression of RAG required signaling through the IL-7R. Consistent with this observation, $\lambda$ light chain transcripts are suppressed by treatment with the IL-7R-blocking antibody. When receptor editing was suppressed, IgG-associated, but not IgM-associated, $\lambda$ chain expression was significantly decreased, strongly suggesting that receptor revision occurs in antigen-activated mature B cells that are undergoing or have completed isotype switching. We propose that upregulation of IL-7R on antigen-activated autoreactive B cells promotes RAG expression and modulates chromatin accessibility for RAG at the light chain locus. IL-7R may also provide a signal to maintain cell viability that allows time for receptor revision to occur.

Furthermore, we showed that receptor revision plays a role in the regulation of an anti-DNA autoantibody response and reduc- tion in accessible DNA leads to a reduction in RAG expression. We confirmed in a second antigen system that soluble antigen can induce receptor revision in antigen-reactive cells and diminish the antigen-specific antibody response. To our knowledge, this is the first direct evidence of receptor revision as a mechanism of tolerance in antigen-activated B cells.

Because the GC response is a source of self-reactive B cells and pathogenic autoantibodies display heavy chain class switching and somatic mutation, understanding tolerance in the antigen-activated B cell and the GC or post-GC B cell is critical to an understanding of many autoimmune diseases. Previous studies have shown that soluble antigen can lead to the deletion of GC B cells or post-GC B cells. We now extend our insight into B cell selection and suggest that antigen-activated $B$ cells that are cross-reactive with self antigen will be positively selected in the GC. After leaving the GC, they, like transitional B cells, remain susceptible to tolerance induction. In the absence of antigenspecific T helper cells or follicular dendritic cells, engagement of BCR with self antigen, such as dsDNA, may induce reexpression of the recombination machinery and receptor revision, resulting in the replacement of self-reactive BCR. In our studies of antibody response to $10-2-\mathrm{KLH}$, we determined that soluble antigen can drive receptor revision. It is possible that antigen need not be soluble to induce receptor revision. For example, we demonstrated that DNase treatment eliminated the antigen that provoked receptor revision in the DWEYS-immunized mice. We do not know if DNase treatment reduces soluble antigen or antigen trapped on a membrane. Tolerance-driven receptor revision is not induced within GCs. There are reasons this may be so. First, recent studies indicate that high activity of PI3 kinase negatively regulates the expression of RAG $(61,62)$. This suggests that RAG may be actively suppressed in GC B cells, since there is an elevation of PI3 kinase activity during $B$ cell activation in the GC response. Second, RAG expression requires a cell-cycle arrest, while GC B cells undergo intensive proliferation.

Just as there was shown to be a temporal window during which soluble antigen can reduce memory B cell formation by inducing apoptosis $(36,63)$, receptor revision also appears to be restricted in time, beginning at day 12 after immunization, peaking at around day 17, and dissipating by day 22 (17). This would allow self antigen to encounter antigen-activated B cells in an environment that is not replete with $\mathrm{T}$ cell help and survival signals from follicular dendritic cells.

In the current study, we have identified a peripheral tolerance checkpoint in antigen-activated B cells, which have undergone $\mathrm{V}$ gene hypermutation and isotype switching and seem to derive from a GC response. Reinduction of RAG in antigen-activated autoreactive early memory B cells requires IL-7R signaling, and the resulting receptor revision contributes to the regulation of autoreactivity. The identification of receptor revision as a tolerance mechanism of antigen-activated B cells will help the pathogenesis of autoimmunity to be understood and provide a potential target for treating autoimmune disease, as it is clear that peripheral tolerance mechanisms are perturbed in autoimmune disease.

\section{Methods}

Mice and immunization. Eight- to ten-week-old female BALB/c and BALB/c $R A G 2^{-/-}$mice were purchased from The Jackson Laboratory or Taconic and housed in specific pathogen-free facilities. Immunization was performed as described (17). In brief, mice were immunized i.p. with $100 \mu \mathrm{l}$ of a 1:1 
emulsion of CFA (Difco), containing $100 \mu \mathrm{g}$ of DWEYSVWLSN peptide on a branched polylysine backbone (DWEYS-MAP; AnaSpec) or ADGSGGRDEMQASMWS conjugated to KLH (10-2-KLH; AnaSpec). These mice were boosted with $100 \mu \mathrm{g}$ of DWEYS-MAP or 10-2-KLH in incomplete Freund's adjuvant (IFA) (Difco) on the date specified in the text (see the legends for Figures 1, 5, and 6). All animal experiments were carried out according to protocols that were reviewed and approved by the Institutional Animal Care and Use Committees of Columbia University and The Feinstein Institute for Medical Research.

Administration of antibody, DNase I, and soluble antigen. For in vivo blocking of IL-7R in mice immunized with DWEYS-MAP, $1 \mathrm{mg}$ of anti-IL-7R $\alpha$ (A7R34; eBioscience) was injected i.v. on days 8, 11, and 14 following the primary immunization. To degrade plasma dsDNA, $500 \mu \mathrm{g}$ of bovine DNase I (Sigma-Aldrich) was injected i.v. from day 9 to day 15 following immunization. Control mice received same amount of heat-inactivated DNase I. In order to examine whether soluble antigen induces RAG expression in antigen-activated B cells, $1 \mathrm{mg}$ of soluble 10-2-BSA or BSA was injected i.v. into 10-2-KLH-immunized mice on days 13,14 , and 15 after the primary challenge. To block IL-7R signaling, $1 \mathrm{mg}$ of anti-IL-7R $\alpha$ was administered to these mice on days 12 and 14, respectively. Control mice received the same amount of rat IgG2a isotype control antibody at the same time points.

Generation of peptide tetramer. DWEYSVWLSN-streptavidin-allophycocyanin (DWEYSVWLSN-streptavidin-APC) tetramers, DWEYSVWLSNstreptavidin-Alexa Fluor 488 tetramer, and DWEYSVWLSN-streptavidinAlexa Fluor 350 tetramer were generated as described (16) with minor modifications. Briefly, 1 volume of biotinylated peptide $(1 \mathrm{mg} / \mathrm{ml})$ was mixed with 4 volumes of streptavidin-Alexa Fluor 488 or -Alexa Fluor 350 $(1 \mathrm{mg} / \mathrm{ml}$; Invitrogen) or with 5 volumes of streptavidin-APC $(1 \mathrm{mg} / \mathrm{ml}$; Invitrogen) and incubated at $4^{\circ} \mathrm{C}$ for a minimum of 3 hours. Before staining, unconjugated peptide was removed from peptide tetramer by gel filtration using a Bio-Gel P-30 column (Bio-Rad). The 10-2 peptide tetramer was generated in the same way as the DWEYS tetramer. Biotinylated peptide was synthesized by AnaSpec.

Reagents and flow cytometry. Spleens were harvested from immunized mice. Single-cell suspensions were prepared as described (17). The following antibodies were used for flow cytometry analysis: peridinin-cholrophyll-protein complex anti-B220 (RA3-6B2; BD Biosciences - Pharmingen), FITC-anti- $\mathrm{K}$ (X36; BD Biosciences - Pharmingen), PE-anti- $\lambda$ (goat polyclonal; SouthernBiotech), FITC-anti-CD127 (A7R34; eBioscience), PE-anti-CD138 (281-2; BD Biosciences), FITC-anti-CD80 (16-10A1; BD Biosciences - Pharmingen), and PE-anti-CD95 (15A7; eBioscience). DWEYS-APC tetramer was used to detect antigen-binding B cells. DAPI was added before flow cytometry to exclude dead cells. Data were acquired by using LSRII flow cytometry (BD Biosciences) and analyzed by using FlowJo software (Tree Star Inc.).

Cell sorting. To sort the tetramer binding populations, splenocytes were prepared from 4-5 mice at day 16 after immunization. T cells, monocytes, and dendritic cells were depleted as previously reported (17). Staining was performed as described above. Immediately after sorting, cells were resuspended in TRIzol (Invitrogen) and frozen at $-140^{\circ} \mathrm{C}$ until RNA isolation. Sorting was performed on a FACSAria Flow Cytometer (Becton Dickinson).

Histology. Spleens were removed from naive mice or mice immunized with DWEYS-MAP or MAP-core and frozen in Tissue-Tek OCT Compound (Sakura). Cryosections of $5-\mu \mathrm{m}$ thickness were prepared and mounted on glass slides. Before staining, slides were fixed with ice-cold acetone for 10 minutes and blocked with PBS, containing 2\% BSA, 5\% goat serum, and $0.2 \%$ Triton X-100 (blocking buffer), for 30 minutes at room temperature. Staining was performed with antibodies in blocking buffer for 1 hour at room temperature. Tetramer staining was performed at $4{ }^{\circ} \mathrm{C}$ overnight. Slides were mounted in Aqua-Poly/Mount
(Polysciences Inc.). Fluorescent microscopy was performed on an AxioCam II microscope (Carl Zeiss Microimaging). Image acquisition was performed with a Hamamatu ORCA-ER camera using the Openlab imaging software (Improvision). According to the fluorochrome used for staining, gray images were colored as follows: red for PE or rhodamine, green for Alexa Fluor 488, blue for Alexa Fluor 350. The following antibodies or reagents were used in staining: Alexa Fluor 488 antiRAG2 (rabbit polyclonal; BD Biosciences - Pharmingen), Alexa Fluor 350 anti-RAG2, PE-anti-B220 (RA3-6B2; BD Biosciences - Pharmingen), DWEYS-Alexa Fluor 488, and rhodamine-peanut agglutinin. Alexa Fluor 488 or 350 anti-RAG2 antibodies were made using Zenon anti-rabbit IgG labeling kits (Invitrogen).

Ig $V$ gene sequence analysis. RNA was isolated from sorted cells by using TRIzOL reagent (Invitrogen). cDNA was prepared using the iScript cDNA Synthesis kit (Bio-Rad). Igk V region gene message was ampli-

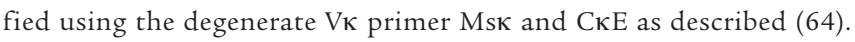
Since the $\mathrm{Tet}^{+} \mathrm{B} 220^{\text {lo }}$ population expresses predominantly IgG1, a pool of forward primers covering the first framework region of heavy chain $\mathrm{V}$ genes (VHfr $1 \mathrm{a}-\mathrm{j}$ ) and a reverse primer at the first domain of $\gamma_{1} \mathrm{C}$ region (65) were used to amplify the IgH V region genes from the $\mathrm{Tet}^{+} \mathrm{B} 220^{\text {lo }}$ cells. PCR product was separated by agarose gel electrophoresis (SigmaAldrich). A single band of proper size was recovered and subjected to TOPO TA cloning (Invitrogen). Plasmid was made from single colonies and subjected to DNA sequencing. The sequences were compared with germline sequences by using the IMGT/V-QUEST (http://imgt.cines.fr). Point mutations between FR1 to CDR 2 regions were counted. Mutations within the first 25 base pairs at the $3^{\prime}$ end of FR1, which is the primer-binding region, were not counted.

Adoptive transfers. Spleens were removed from naive 10- to 12 -week-old $\mathrm{BALB} / \mathrm{c}$ mice. A total of $4 \times 10^{7}$ splenocytes or $2 \times 10^{7}$ isolated lymphocytes were injected i.v. into $R A G 2^{-/-}$mice. Recipient mice were immunized according to the protocol described.

qPCR. RNA isolation and cDNA preparation were described above. qPCR was performed by using an ABI 7900 (Applied Biosystems) and analyzed using SDS version 2.3 (Applied Biosystems). Applied Biosystems Gene Expression Assays were used, and the reactions were performed using the TaqMan Universal PCR Master Mix (Applied Biosystems) in $20 \mu \mathrm{l}$ final volume. Standard curves were made for each experiment using total spleen and BM cDNA. Relative template concentration was determined from the standard curve by using Cts determined by the SDS software (Applied Biosystems). The RNA polymerase $2 \mathrm{a}$ (polr2a) was used as an internal control. All primers used spanned an intron/exon border. Applied Biosystems Primer IDs were as follows: Ig $\mu, \mathrm{Mm01718956 \_ m1;} \mathrm{Ig \gamma ,} \mathrm{custom}$ designed primers; RAG1, Mm01270936_m1; RAG2, Mm00501300_m1; IL-7R $\alpha$ (CD127), Mm00434295_m1; AID, Mm0050774_m1; Blimp1, Mm00476128; Xbp1, Mm00457359_m1; Igh, Mm01611614_m1; RNA pol2a, Mm00839493_m1.

Peptide ELISA. EIA/RIA high-binding 96-well plates (Costar) were coated with $50 \mu \mathrm{l}$ DWEYS-MAP or 10-2-ovalbumin at $15 \mu \mathrm{g} / \mathrm{ml}$ in PBS and incubated overnight at $4^{\circ} \mathrm{C}$. Plates were blocked with $150 \mu$ l of $1 \%$ BSA in PBS (HyClone) at $37^{\circ} \mathrm{C}$ for 1 hour. Fifty microliters of diluted sera in $1 \%$ BSA were added to the plates for 1 hour at $37^{\circ} \mathrm{C}$. Plates were then washed with PBSTween, and $50 \mu$ l of alkaline phosphatase-conjugated (AP-conjugated) goat anti-mouse IgM or IgG (SouthernBiotech) diluted 1:1,000 in 1\% BSA/PBS was added to the plates for 1 hour at $37^{\circ} \mathrm{C}$. After washing with PBS-Tween, $100 \mu \mathrm{l}$ of $p$-nitrophenyl phosphate solution (Sigma-Aldrich) was added and the OD was monitored at $405 \mathrm{~nm}$, using a Titer-Tek Multiscan ELISA reader (Titertek). To determine how much IgM or IgG was bound to the plate, a standard curve was generated with mouse IgM or IgG (SouthernBiotech) on anti-IgM or anti-IgG adsorbed plates. 
dsDNA ELISA. Calf-thymus DNA (Calbiochem-Novabiochem) was dissolved in PBS, sonicated, and filtered through a $0.45-\mu \mathrm{m}$ nitrocellulose Millex syringe filter (Millipore) to produce dsDNA in 10- to 20-kbp fragments. Fifty microliters of $100 \mu \mathrm{g} / \mathrm{ml}$ dsDNA was used to coat EIA/RIA high-binding 96-well plates (Costar) overnight at $37^{\circ} \mathrm{C}$. The remainder of the assay was performed as described for the peptide ELISA. To determine how much IgM or IgG was bound to the plate, a standard curve was generated, using mouse IgM or IgG (SouthernBiotech) on anti-IgM or anti-IgG adsorbed plates.

$\lambda$ light chain ELISA. To measure the serum levels of $\lambda$ light chain, the Costar 96-well plates were coated with goat anti-mouse IgM or IgG at $4^{\circ} \mathrm{C}$ overnight. Plates were blocked with $1 \% \mathrm{BSA} / \mathrm{PBS}$ for 1 hour at room temperature. Diluted sera were added to the plate and incubated for 1 hour at room temperature. AP-conjugated goat anti-mouse $\lambda$ light chain was used as the detection antibody. The rest of the assay was performed as described above. A standard curve was generated, using mouse $\operatorname{IgM}-\lambda$ (11E10; SouthernBiotech) or IgG1- $\lambda$ (A111-3; BD Biosciences - Pharmingen) for quantitation of IgM or IgG associated with $\lambda$ light chain.
Statistics. $P$ values were determined by 2 -way ANOVA or Student's 2 -tailed $t$ test as specified in the text. $P$ values of less than 0.05 were considered significant.

\section{Acknowledgments}

The authors thank Stella Stefanova for assistance with flow cytometry. This study was supported by grants from NIH (PO1 AI51392 and NIH-R37 ARO 32371 to B. Diamond).

Received for publication March 13, 2008, and accepted in revised form June 11, 2008.

Address correspondence to: Betty Diamond, Center for Autoimmune and Musculoskeletal Disease, The Feinstein Institute for Medical Research, North Shore LIJ Health System, 350 Community Drive, Manhasset, New York 11030, USA. Phone: (516) 562-3830; Fax: (516) 562-2953; E-mail: bdiamond@nshs.edu.
1. Nossal, G.J. 1983. Cellular mechanisms of immunologic tolerance. Annu. Rev. Immunol. 1:33-62.

2. Goodnow, C.C. 1996. Balancing immunity and tolerance: deleting and tuning lymphocyte repertoires. Proc. Natl. Acad. Sci. U. S. A. 93:2264-2271.

3. Radic, M.Z., Erikson, J., Litwin, S., and Weigert, M. 1993. B lymphocytes may escape tolerance by revising their antigen receptors. J. Exp. Med. 177:1165-1173.

4. Tiegs, S.L., Russell, D.M., and Nemazee, D. 1993 Receptor editing in self-reactive bone marrow B cells. J. Exp. Med. 177:1009-1020.

5. Goodnow, C.C., Adelstein, S., and Basten, A. 1990. The need for central and peripheral tolerance in the B cell repertoire. Science. 248:1373-1379.

6. Kilmon, M.A., Rutan, J.A., Clarke, S.H., and Vilen, B.J. 2005. Low-affinity, Smith antigen-specific B cells are tolerized by dendritic cells and macrophages. J. Immunol. 175:37-41.

7. Mackay, M., et al. 2006. Selective dysregulation of the FcgammaIIB receptor on memory B cells in SLE. J. Exp. Med. 203:2157-2164.

8. Han, S., Zheng, B., Schatz, D.G., Spanopoulou, E., and Kelsoe, G. 1996. Neoteny in lymphocytes: Rag1 and Rag2 expression in germinal center B cells. Science. 274:2094-2097.

9. Hikida, M., et al. 1996. Reexpression of RAG-1 and RAG-2 genes in activated mature mouse B cells. Science. 274:2092-2094.

10. Han, S., Zheng, B., Dal Porto, J., and Kelsoe, G. 1995. In situ studies of the primary immune response to (4-hydroxy-3-nitrophenyl)acetyl. IV. Affinity-dependent, antigen-driven B cell apoptosis in germinal centers as a mechanism for maintaining self-tolerance. J. Exp. Med. 182:1635-1644.

11. Girschick, H.J., Grammer, A.C., Nanki, T., Vazquez, E., and Lipsky, P.E. 2002. Expression of recombination activating genes 1 and 2 in peripheral $B$ cells of patients with systemic lupus erythematosus. Arthritis Rheum. 46:1255-1263.

12. Morbach, H., Singh, S.K., Faber, C., Lipsky, P.E., and Girschick, H.J. 2006. Analysis of RAG expression by peripheral blood CD5 + and CD5- B cells of patients with childhood systemic lupus erythematosus. Ann. Rheum. Dis. 65:482-487.

13. Zhang, Z., Wu, X., Limbaugh, B.H., and Bridges, S.L., Jr. 2001. Expression of recombination-activating genes and terminal deoxynucleotidyl transferase and secondary rearrangement of immunoglobulin kappa light chains in rheumatoid arthritis synovial tissue. Arthritis Rheum. 44:2275-2284.

14. Girschick, H.J., Grammer, A.C., Nanki, T., Mayo, M., and Lipsky, P.E. 2001. RAG1 and RAG2 expression by $B$ cell subsets from human tonsil and peripheral blood. J. Immunol. 166:377-386.
15. Putterman, C., and Diamond, B. 1998. Immunization with a peptide surrogate for double-stranded DNA (dsDNA) induces autoantibody production and renal immunoglobulin deposition. J. Exp. Med. 188:29-38.

16. Newman, J., Rice, J.S., Wang, C., Harris, S.L., and Diamond, B. 2003. Identification of an antigenspecific B cell population. J. Immunol. Methods. 272:177-187.

17. Rice, J.S., Newman, J., Wang, C., Michael, D.J., and Diamond, B. 2005. Receptor editing in peripheral B cell tolerance. Proc. Natl. Acad. Sci. U. S. A. 102:1608-1613.

18. Khalil, M., Inaba, K., Steinman, R., Ravetch, J., and Diamond, B. 2001. T cell studies in a peptideinduced model of systemic lupus erythematosus. J. Immunol. 166:1667-1674.

19. MacLennan, I.C., et al. 2003. Extrafollicular antibody responses. Immunol. Rev. 194:8-18.

20. Paus, D., et al. 2006. Antigen recognition strength regulates the choice between extrafollicular plasma cell and germinal center B cell differentiation. J. Exp. Med. 203:1081-1091.

21. Shapiro-Shelef, M., and Calame, K. 2005. Regulation of plasma-cell development. Nat. Rev. Immunol. 5:230-242.

22. Calame, K. 2006. Transcription factors that regulate memory in humoral responses. Immunol. Rev 211:269-279

23. Anderson, S.M., Tomayko, M.M., Ahuja, A., Haberman, A.M., and Shlomchik, M.J. 2007. New markers for murine memory B cells that define mutated and unmutated subsets. J. Exp. Med. 204:2103-2114.

24. McHeyzer-Williams, L.J., Cool, M., and McHeyzer-Williams, M.G. 2000. Antigen-specific B cell memory: expression and replenishment of a novel b220(-) memory b cell compartment. J. Exp. Med. 191:1149-1166.

25. Kitamura, D., Roes, J., Kühn, R., and Rajewsky, K. 1991. A B cell-deficient mouse by targeted disruption of the membrane exon of the immunoglobulin mu chain gene. Nature. 350:423-426.

26. Hikida, M., et al. 1998. Expression of recombination activating genes in germinal center B cells: involvement of interleukin 7 (IL-7) and the IL-7 receptor. J. Exp. Med. 188:365-372.

27. Billips, L.G., et al. 1995. Immunoglobulin recombinase gene activity is modulated reciprocally by interleukin 7 and CD19 in B cell progenitors. J. Exp. Med. 182:973-982.

28. Ben-Yehuda, A., et al. 1999. Activation of the recombination activating gene 1 (RAG-1) transcript in bone marrow of senescent C57BL/ 6 mice by recombinant interleukin-7. J. Gerontol. A Biol. Sci. Med. Sci. 54:B143-B148.
29. Huang, J., and Muegge, K. 2001. Control of chromatin accessibility for $\mathrm{V}(\mathrm{D}) \mathrm{J}$ recombination by interleukin-7. J. Leukoc. Biol. 69:907-911.

30. Crompton, T., Outram, S.V., Buckland, J., and Owen, M.J. 1997. A transgenic T cell receptor restores thymocyte differentiation in interleukin-7 receptor alpha chain-deficient mice. Eur. J. Immunol. 27:100-104.

31. Schluns, K.S., Kieper, W.C., Jameson, S.C., and Lefrancois, L. 2000. Interleukin-7 mediates the homeostasis of naive and memory CD8 T cells in vivo. Nat. Immunol. 1:426-432.

32. Kondrack, R.M., et al. 2003. Interleukin 7 regulates the survival and generation of memory CD 4 cells. J. Exp. Med. 198:1797-1806.

33. Franchimont, D., et al. 2002. Positive effects of glucocorticoids on $\mathrm{T}$ cell function by up-regulation of IL-7 receptor alpha. J. Immunol. 168:2212-2218.

34. Meffre, E., et al. 1998. Antigen receptor engagement turns off the $V(D) J$ recombination machinery in human tonsil B cells. J. Exp. Med. 188:765-772.

35. Putterman, C., Deocharan, B., and Diamond, B. 2000. Molecular analysis of the autoantibody response in peptide-induced autoimmunity. J. Immunol. 164:2542-2549.

36. Harris, S.L., Park, M.K., Nahm, M.H., and Diamond, B. 2000. Peptide mimic of phosphorylcholine, a dominant epitope found on Streptococcus pneumoniae. Infect. Immun. 68:5778-5784.

37. Pulendran, B., Kannourakis, G., Nouri, S., Smith, K.G., and Nossal, G.J. 1995. Soluble antigen can cause enhanced apoptosis of germinal-centre B cells. Nature. 375:331-334.

38. Shokat, K.M., and Goodnow, C.C. 1995. Antigeninduced B-cell death and elimination during germinal-centre immune responses. Nature. 375:334-338.

39. Goodnow, C.C., Crosbie, J., Jorgensen, H., Brink, R.A., and Basten, A. 1989. Induction of self-tolerance in mature peripheral B lymphocytes. Nature. 342:385-391.

40. Russell, D.M., et al. 1991. Peripheral deletion of self-reactive B cells. Nature. 354:308-311.

41. Tsuiji, M., et al. 2006. A checkpoint for autoreactivity in human IgM+ memory B cell development. J. Exp. Med. 203:393-400.

42. William, J., Euler, C., Primarolo, N., and Shlomchik, M.J. 2006. B cell tolerance checkpoints that restrict pathways of antigen-driven differentiation. J. Immunol. 176:2142-2151.

43. Culton, D.A., et al. 2006. Early preplasma cells define a tolerance checkpoint for autoreactive B cells. J. Immunol. 176:790-802.

44. Diamond, B., and Scharff, M.D. 1984. Somatic mutation of the T15 heavy chain gives rise to an antibody with autoantibody specificity. Proc. Natl. 
Acad. Sci. U. S. A. 81:5841-5844.

45. Ray, S.K., Putterman, C., and Diamond, B. 1996. Pathogenic autoantibodies are routinely generated during the response to foreign antigen: a paradigm for autoimmune disease. Proc. Natl. Acad. Sci. U.S. A. 93:2019-2024.

46. Kuo, P., Bynoe, M., and Diamond, B. 1999. Crossreactive $B$ cells are present during a primary but not secondary response in BALB/c mice expressing a bcl-2 transgene. Mol. Immunol. 36:471-479.

47. Linton, P.J., Rudie, A., and Klinman, N.R. 1991. Tolerance susceptibility of newly generating memory B cells. J. Immunol. 146:4099-4104.

48. Jankovic, M., Casellas, R., Yannoutsos, N., Wardemann, H., and Nussenzweig, M.C. 2004. RAGs and regulation of autoantibodies. Annu. Rev. Immunol. 22:485-501.

49. Gartner, F., Alt, F.W., Monroe, R.J., and Seidl, K.J. 2000. Antigen-independent appearance of recombination activating gene (RAG)-positive bone marrow B cells in the spleens of immunized mice. J. Exp. Med. 192:1745-1754.

50. Nagaoka, H., Gonzalez-Aseguinolaza, G., Tsuji, M., and Nussenzweig, M.C. 2000. Immunization and infection change the number of recombination activating gene (RAG)-expressing B cells in the periphery by altering immature lymphocyte production. J. Exp. Med. 191:2113-2120.

51. Wilson, P.C., et al. 2000. Receptor revision of immunoglobulin heavy chain variable region genes in normal human B lymphocytes. J. Exp. Med. 191:1881-1894.

52. Igarashi, H., et al. 2001. Localization of recombination activating gene 1 /green fluorescent protein (RAG1/GFP) expression in secondary lymphoid organs after immunization with T-dependent antigens in rag1/gfp knockin mice. Blood. 97:2680-2687.

53. Meru, N., Jung, A., Baumann, I., and Niedobitek, G. 2002. Expression of the recombination-activating genes in extrafollicular lymphocytes but no apparent reinduction in germinal center reactions in human tonsils. Blood. 99:531-537.

54. Papavasiliou, F., et al. 1997. V(D)J recombination in mature B cells: a mechanism for altering antibody responses. Science. 278:298-301.

55. Hillion, S., Saraux, A., Youinou, P., and Jamin, C. 2005. Expression of RAGs in peripheral B cells outside germinal centers is associated with the expression of CD5. J. Immunol. 174:5553-5561.

56. Han, S., et al. 1997. V(D)J recombinase activity in a subset of germinal center B lymphocytes. Science. 278:301-305.

57. Hertz, M., Kouskoff, V., Nakamura, T., and Nemazee, D. 1998. V(D)J recombinase induction in splenic B lymphocytes is inhibited by antigenreceptor signalling. Nature. 394:292-295.

58. Yu, W., et al. 1999. Continued RAG expression in late stages of B cell development and no apparent reinduction after immunization. Nature. 400:682-687. 59. Monroe, R.J., et al. 1999. RAG2:GFP knockin mice reveal novel aspects of RAG2 expression in primary and peripheral lymphoid tissues. Immunity. 11:201-212.

60. Kuwata, N., Igarashi, H., Ohmura, T., Aizawa, S., and Sakaguchi, N. 1999. Cutting edge: absence of expression of RAG1 in peritoneal B-1 cells detected by knocking into RAG1 locus with green fluorescent protein gene. J. Immunol. 163:6355-6359.

61. Llorian, M., Stamataki, Z., Hill, S., Turner, M., and Martensson, I.L. 2007. The PI3K p110delta is required for down-regulation of RAG expression in immature B cells. J. Immunol. 178:1981-1985.

62. Verkoczy, L., et al. 2007. Basal B cell receptor-directed phosphatidylinositol 3-kinase signaling turns off RAGs and promotes B cell-positive selection. J. Immunol. 178:6332-6341.

63. Nossal, G.J., Karvelas, M., and Pulendran, B. 1993. Soluble antigen profoundly reduces memory B-cell numbers even when given after challenge immunization. Proc. Natl. Acad. Sci. U. S. A. 90:3088-3092.

64. Oberdoerffer, P., Novobrantseva, T.I., and Rajewsky, K. 2003. Expression of a targeted lambda 1 light chain gene is developmentally regulated and independent of Ig kappa rearrangements. J. Exp. Med. 197:1165-1172.

65. Kettleborough, C.A., Saldanha, J., Ansell, K.H., and Bendig, M.M. 1993. Optimization of primers for cloning libraries of mouse immunoglobulin genes using the polymerase chain reaction. Eur. J. Immunol. 23:206-211. 